\title{
Unitarity in Reissner-Nordström background: striding away from information loss
}

\author{
Arpit Das $^{\mathrm{a}}$, Narayan Banerjee ${ }^{\mathrm{b}}$ \\ Department of Physical Sciences, Indian Institute of Science Education and Research Kolkata, Mohanpur, West Bengal 741246, India
}

Received: 1 May 2019 / Accepted: 25 May 2019 / Published online: 5 June 2019

(C) The Author(s) 2019

\begin{abstract}
We have shown analytically that radiation from a collapsing shell which leads to a charged black hole, whose exterior is described by the Reissner-Nordström metric (and hence the background spacetime is non-globally hyperbolic), is processed with a unitary evolution. For the analysis, we have used the Wheeler-deWitt formalism which in turn gave rise to a Schrödinger-like wave equation. We showed the existence of unitarity by proving that the trace of the squared density matrix of the outgoing radiation, from a quantized massless scalar field, is unity and that the conservation of probability holds for the wave function of the system.
\end{abstract}

\section{Introduction}

The information loss paradox, since its inception [1,2], has been open to many diverse interpretations [3-5]. A traditional interpretation is: when a Schwarzschild black hole completely evaporates due to Hawking radiation the resultant spacetime becomes non-globally hyperbolic and hence quantum processes in such a spacetime would be non-unitary $[6,7]$. This, as traditionally argued, would lead to information loss. On the contrary, we show that even in a nonglobally hyperbolic spacetime, for instance in a ReissnerNordström (RN) background, unitarity can be achieved. We do this by adopting the Wheeler-deWitt formalism as in Refs. $[8,9]$. In quantum mechanics, the evolution of pure states to mixed states may be understood as a non-unitary evolution. Recently, it has been shown by Saini and Stojkovic [10], in the context of a Schwarzchild black hole (and hence in a globally hyperbolic spacetime), that the evolution of quantum fields is actually unitary! They achieved this by showing that the traces of the density matrix and its square are unity for both the initial and the final states with proper normalization. However, they accomplished this by numerical

\footnotetext{
a e-mail: ad13ms118@iiserkol.ac.in

b e-mail: narayan@iiserkol.ac.in
}

estimates (over a period of finite proper time) which depend on the accuracy of the method and the reliability has to be ascertained carefully.

The present work shows for the first time, analytically, that the process of black hole radiation, even in a non-globally hyperbolic background, is unitary. This is proved using the consideration of density matrix, as in Ref. [10], but analytically. So the result is far more robust now. We further show, from quite an independent calculation, that the conservation of probability holds good in this process confirming an unitary evolution! Thus the result is now confirmed from two independent lines of approach. Furthermore, this is worked out for a non-globally hyperbolic background unlike the globally hyperbolic scenario as investigated in Ref. [10].

We work with an RN metric that includes an electric charge. The Schwarzchild case, considered in Ref. [10], is recovered as a special case by putting the charge $Q=0$. This generalization actually has important consequences. One can recover the extremal $\mathrm{RN}$ results by setting $|Q|=M$, where $M$ is the mass of the black hole. So, this work might have a profound significance in the context of string theory where an extremal RN black hole is a mainstay.

\section{The model}

The model we will be studying comprises an infinitesimally thin collapsing charged spherical shell, with background metric $g_{\mu \nu}$ and a massless scalar field $\Phi$ whose dynamics we are interested in. The massless scalar field is assumed to couple to the gravitational field (originating from the presence of a non-trivial background metric), but not directly to the shell. We also have an asymptotic observer, sitting at the future null infinity, who is just there to register the outgoing flux with a detector and hence by assumption has very little or no interaction with "shell-metric-scalar" system. Also, the observer is assumed not to significantly affect the evolution of the sys- 
tem and similarly for the system vis-a-vis the observer. The action for the whole system is then given by [8],

$$
\begin{aligned}
S_{\text {tot }}= & \int d^{4} x \sqrt{-g}\left[-\frac{\mathcal{R}}{16 \pi}+\frac{1}{2}\left(\partial_{\mu} \Phi\right)^{2}\right]-\sigma \int d^{3} \xi \sqrt{-\gamma} \\
& +S_{\text {obs }},
\end{aligned}
$$

where the first term is the Einstein-Hilbert term for the background metric $g_{\mu \nu}$, the second term is the action for the massless scalar field, the third term is shell's action in terms of its world-volume coordinates $\xi^{a}(a=0,1,2)$, the shell's ten$\operatorname{sion} \sigma$ (or, shell's proper energy density per unit surface area) and the shell's induced world-volume metric $\gamma_{a b}$, which is given by,

$\gamma_{a b}=g_{\mu \nu} \partial_{a} X^{\mu} \partial_{b} X^{\nu}$

where $X^{\mu}\left(\xi^{a}\right)$ gives the location of the shell. The Roman indices run over the internal world-volume coordinates $\xi^{a}(a=0,1,2)$ while the Greek indices run over the usual spacetime coordinates.

Lastly, $S_{o b s}$ is the action for the observer.

\section{Spacetime foliation- $\mathrm{RN}$ coordinates}

We consider that the mass and the charge is confined in an infinitesimally thin shell, so that for an exterior observer the distribution is spherical, whereas the inside of the shell is empty given by the Minkowski metric. The exterior of the shell is described by a RN metric and its uniqueness is guaranteed by the charged version of Birkhoff's theorem $[12,13]$. Thus, we have,

$$
\begin{aligned}
& d s_{\text {out }}^{2}=-\left(1-\frac{2 M}{r}+\frac{Q^{2}}{r^{2}}\right) d t^{2} \\
&+\left(1-\frac{2 M}{r}+\frac{Q^{2}}{r^{2}}\right)^{-1} d r^{2}+r^{2} d \Omega_{2}^{2}, \\
& d s_{\text {in }}^{2}=- d T^{2}+d r^{2}+r^{2} d \Omega_{2}^{2}, \\
& d s_{\text {on-shell }}^{2}=-d \tau^{2}+r^{2} d \Omega_{2}^{2},
\end{aligned}
$$

for $r>R(t), r<R(t)$ and $r=R(t)$ respectively. $r$ is simply the radial coordinate and so $r=R(t)$ describes the shell. Furthermore, $R:=R(t), t, T$ and $\tau$ are the radius of the shell, time coordinate of the exterior observer, the time coordinate inside the shell and the proper time on the shell respectively. $d \Omega_{2}^{2}$ is the usual $S^{2}$ metric.

One important thing to note here is that since RN coordinates leads to a coordinate singularity, at $R=R_{H}:=$ $M+\sqrt{M^{2}-Q^{2}}$ (the event horizon), we might be in trouble using this for our analysis. However, note that for an asymptotic observer the event horizon is an infinitely red shifted surface. Hence, the observer can only notice the collapse of the shell approaching its event horizon in infinite time as per his time $t$. So, our analysis happens upto this limit which is relevant from an asymptotic viewpoint and $\mathrm{RN}$ coordinates are well behaved upto this limit, that is just outside the event horizon.

We consider timelike unit vectors $u^{\alpha}:=\frac{d x_{\text {out }}^{\alpha}}{d \tau}$ and $v^{\alpha}:=\frac{d x_{\text {in }}^{\alpha}}{d \tau}$, for $d s_{\text {out }}^{2}$ and $d s_{\text {in }}^{2}$ respectively. From their normalization, that is, $u^{\alpha} u_{\alpha}=-1$ and $v^{\alpha} v_{\alpha}=-1$, one obtains, at $r=R(t), t_{\tau}=\frac{\sqrt{D+R_{\tau}^{2}}}{D}, T_{\tau}=\sqrt{1+R_{\tau}^{2}}$ and $T_{t}=\sqrt{D-(1-D) \frac{R_{t}^{2}}{D}}$. In the above expressions, a subscript indicates a differentiation w.r.t. that particular coordinate. $x_{\text {out }}^{\alpha}$ and $x_{i n}^{\alpha}$ are the coordinates pertaining to $d s_{\text {out }}^{2}$ and $d s_{i n}^{2}$ respectively. Also, $D:=1-\frac{2 M}{R(t)}+\frac{Q^{2}}{R(t)^{2}}$.

\section{Mass of the shell}

Using Israel's formulation [14], the mass $M$ of the shell is (see also [15]),

$M=4 \pi \sigma R^{2}\left[\sqrt{1+R_{\tau}^{2}}-2 \pi \sigma R\right]+\frac{Q^{2}}{2 R}$,

We show below that $M$ is a constant of motion. So, there is no conflict with the fact that $M$ is a constant of integration in the metric and is identified as the mass of the shell. Using the results given in [16], one can write,

$$
\frac{R_{\tau \tau}}{\alpha}=\frac{Q^{2}}{8 \pi \sigma R^{4}}+6 \pi \sigma-\frac{2 \alpha}{R},\left(\text { where, } \alpha:=\sqrt{1+R_{\tau}^{2}}\right) \text {. }
$$

Then, using $e q^{n} s$ (6) and (7),

$$
\begin{aligned}
M_{\tau}= & R_{\tau}\left[8 \pi \sigma R(\alpha-2 \pi \sigma R)-\frac{Q^{2}}{2 R^{2}}\right] \\
& +R_{\tau}\left[4 \pi \sigma R^{2}\left(\frac{Q^{2}}{8 \pi \sigma R^{4}}-\frac{2 \alpha}{R}+6 \pi \sigma-2 \pi \sigma\right)\right]=0 .
\end{aligned}
$$

So, $M$ is a constant of motion.

Interpretations of $M$ can be looked at as follows. Suppose $R_{\tau}=0$ in $e q^{n}$ (6) (a static shell), then,

$M_{\text {static }}=4 \pi \sigma R^{2}-8 \pi^{2} \sigma^{2} R^{3}+\frac{Q^{2}}{2 R}$,

where the three terms represent the rest mass term, gravitational self-interaction term and the electrostatic selfinteraction term respectively. If $R_{\tau} \neq 0$, then the term with $\sqrt{1+R_{\tau}^{2}}$ in $e q^{n}$ (6) is the kinetic energy term. To 
get more intuition out of this, let us look at $M$ in the nonrelativistic limit (where $R_{\tau}<<1$ ). By identifying constant mass $M_{0}:=4 \pi \sigma R^{2}$, we have from $e q^{n}(6)$,

$M_{n o n-r e l}=M_{0}+\frac{p^{2}}{2 M_{0}}-\frac{M_{0}^{2}}{2 R}+\frac{Q^{2}}{2 R}$,

where $p:=\frac{1}{2} M_{0} R_{\tau}^{2}$ is the momentum of the particle with constant mass $M_{0}$ moving in a gravitational and electrostatic potential. Here the second term represents the kinetic energy. One can clearly identify $e q^{n}(9)$ as the Hamiltonian of a nonrelativistic particle moving under the influence of a gravitational and electrostatic potential (see [16]). From relativistic perspectives, note that (see [9]),

$M_{r e l}=\frac{M_{0}}{\sqrt{1-R_{T}^{2}}}-\frac{M_{0}^{2}}{2 R}+\frac{Q^{2}}{2 R}$,

which is the Hamiltonian of a relativistic particle with rest mass $M_{0}$ moving in a gravitational and electrostatic potential.

Since, we have shown that $M$ is a constant of motion, by the above interpretation of $M$, we have the following identification,

$\mathcal{H}_{\text {shell }} \equiv M$

where $H_{\text {shell }}$ is the Hamiltonian of the shell to be treated classically.

\section{Action for the shell}

The form of the action, for the shell, is taken as

$S_{\text {shell }}=-\int d T\left[4 \pi \sigma R^{2}\left[\sqrt{1-R_{T}^{2}}-2 \pi \sigma R\right]+\frac{Q^{2}}{2 R}\right]$.

The corresponding Lagrangian yields the conjugate momentum, for the shell, as,

$\Pi_{\text {shell }}=\frac{\partial \mathcal{L}_{\text {shell }}}{\partial R_{T}}=4 \pi \sigma R^{2}\left(\frac{R_{T}}{\sqrt{1-R_{T}^{2}}}\right)$.

Then, the Hamiltonian is,

$$
\begin{aligned}
\mathcal{H}_{\text {shell }} & =\Pi_{\text {shell }} R_{T}-\mathcal{L}_{\text {shell }} \\
& =4 \pi \sigma R^{2}\left[\sqrt{1+R_{\tau}^{2}}-2 \pi \sigma R\right]+\frac{Q^{2}}{2 R} .
\end{aligned}
$$

This matches with $M$ as expressed in $e q^{n}$ (6). So, the action in $e q^{n}$ (12) is consistent [since, this action gives the correct
$\mathcal{H}_{\text {shell }}$ as expressed in $e q^{n}$ (11)]. In terms of time $t$, (using the expression for $T_{t}$ ) $S_{\text {shell }}$ becomes,

$$
\begin{aligned}
S_{\text {shell }}= & -\int d t \\
& \left.+\int \pi \sigma R^{2}\left[\sqrt{D-\frac{R_{t}^{2}}{D}}\right]\right] \\
& +\int d t\left[4 \pi \sigma R^{2}\left[2 \pi \sigma R \sqrt{D-\frac{1-D}{D} R_{t}^{2}}\right]\right] \\
& -\int d t \\
& {\left[\frac{Q^{2}}{2 R} \sqrt{D-\frac{1-D}{D} R_{t}^{2}}\right] . }
\end{aligned}
$$

The conjugate momentum and Hamiltonian (in terms of $t$ ) are,

$$
\begin{gathered}
\Pi_{\text {shell }}=\frac{\partial \mathcal{L}_{\text {shell }}}{\partial R_{t}}=\frac{4 \pi \sigma R^{2} R_{t}}{\sqrt{D}} \\
{\left[\frac{1}{\sqrt{D^{2}-R_{t}^{2}}}-\frac{2 \pi \sigma R(1-D)}{\sqrt{D^{2}-(1-D) R_{t}^{2}}}\right]} \\
+\frac{4 \pi \sigma R^{2} R_{t}}{\sqrt{D}}\left[\frac{Q^{2}(1-D)}{8 \pi \sigma R^{3} \sqrt{D^{2}-(1-D) R_{t}^{2}}}\right] \\
\mathcal{H}_{\text {shell }}=\Pi_{\text {shell }} R_{t}-\mathcal{L}_{\text {shell }} \\
=4 \pi \sigma D^{3 / 2} R^{2}\left[\frac{1}{\sqrt{D^{2}-R_{t}^{2}}}-\frac{2 \pi \sigma R}{\sqrt{D^{2}-(1-D) R_{t}^{2}}}\right] \\
+4 \pi \sigma D^{3 / 2} R^{2}\left[\frac{Q^{2}}{8 \pi \sigma R^{3} \sqrt{D^{2}-(1-D) R_{t}^{2}}}\right]
\end{gathered}
$$

\section{Incipient limit}

The incipient limit, $R \rightarrow R_{H}$, is the limit when the radius of the shell approaches the event horizon,

$R_{H}=M+\sqrt{M^{2}-Q^{2}}$.

From $e q^{n}$ (16) and $e q^{n}$ (17) we note that, in the incipient limit,

$$
\begin{aligned}
\Pi_{\text {shell }} & =\frac{4 \pi \mu R^{2} R_{t}}{\sqrt{D} \sqrt{D^{2}-R_{t}^{2}}} \\
\mathcal{H}_{\text {shell }} & =\frac{4 \pi D^{3 / 2} \mu R^{2}}{\sqrt{D^{2}-R_{t}^{2}}}
\end{aligned}
$$


where, $\mu:=\sigma\left(1-2 \pi \sigma R_{H}+\frac{Q^{2}}{8 \pi \sigma R_{H}^{3}}\right)$. So we have,

$\mathcal{H}_{\text {shell }}=\left[\left(D \Pi_{\text {shell }}\right)^{2}+D\left(4 \pi \mu R^{2}\right)^{2}\right]^{1 / 2} \equiv\left[q^{2}+m^{2}\right]^{1 / 2}$,

where $q^{2}:=\left(D \Pi_{\text {shell }}\right)^{2}$ and $m^{2}:=D\left(4 \pi \mu R^{2}\right)^{2}$.

Equation (21) shows that $\mathcal{H}_{\text {shell }}$ is the Hamiltonian of a relativistic particle with a position dependent mass. So, that is how the shell behaves in the incipient limit. Let us now show that in this limit also, $\mathcal{H}_{\text {shell }}$ is a constant of motion. Since, $\frac{d \mathcal{H}_{\text {shell }}}{d \tau}=\frac{\partial \mathcal{H}_{\text {shell }}}{\partial \tau}$, we have,

$\frac{d}{d \tau}\left(4 \pi \mu \frac{D^{3 / 2} R^{2}}{\sqrt{D^{2}-R_{t}^{2}}}\right)=0$

leading to, $\frac{D^{3 / 2} R^{2}}{\sqrt{D^{2}-R_{t}^{2}}}=\frac{\mathcal{H}_{\text {shell }}}{4 \pi \mu}=: h$ (a constant),

(as $\tau$ doesn't appear explicitly in $\mathcal{H}_{\text {shell }}$ ).

We can arrive at these expressions independently using an alternative approach (see Appendix)

Classically, we have from $e q^{n}(22)$ and $T_{t}$,

$R_{t}= \pm D \sqrt{1-\frac{D R^{4}}{h^{2}}} \approx \pm D\left(1-\frac{1}{2} \frac{D R^{4}}{h^{2}}\right) \approx \pm D$,

$\left(\right.$ as $\left.R \rightarrow R_{H}\right)$,

$T_{t}=D \sqrt{1+(1-D) \frac{R^{4}}{h^{2}}}$,

where solving $e q^{n}$ (23) in terms of $t$ will give us the classical behaviour of the shell as the event horizon is approached.

\section{Non-extremal case}

The horizons (outer and inner) of the charged shell are given by,

$R_{ \pm}=M \pm \sqrt{M^{2}-Q^{2}}$.

$D$ can be written as,

$D=\left(1-\frac{R_{+}}{R}\right)\left(1-\frac{R_{-}}{R}\right)=: D_{+} D_{-}$.

In the incipient limit, $D \rightarrow 0$ and hence, $D_{+} \rightarrow 0$ (as $R(t)$ approaches the event horizon $R_{H}=R_{+}$). Thus, for $D_{+} \rightarrow$ 0 , we have $D_{-} \rightarrow 1-\frac{R_{-}}{R_{+}}=:\left.D_{-}\right|_{i}$. Then, in this limit,
$R_{t} \approx \pm D= \pm D_{+} D_{-}$. Solving for $R(t)$ we get [from $e q^{n}$ (23) to $\left.e q^{n}(25)\right]$,

$\pm 1=\frac{1}{D_{-}} \frac{R}{R-R_{+}} \frac{d R}{d t} \approx \frac{1}{\left.D_{-}\right|_{i}} \frac{R_{+}}{R-R_{+}} \frac{d R}{d t}$

(upto leading order)

integrating w.r.t.t, $R_{+} \ln \left(\frac{R_{f}-R_{+}}{R_{0}-R_{+}}\right)= \pm\left. t_{f} D_{-}\right|_{i}$

$\left(R_{0}:=R(0)\right.$ and $\left.R_{f}:=R\left(t_{f}\right)\right)$

and thus, $R_{f}=R_{+}+\left(R_{0}-R_{+}\right) e^{ \pm\left. D_{-}\right|_{i} t_{f} / R_{+}}$,

where the lower limit of integration w.r.t. $t$ is $t=0$ and the upper limit is $t=t_{f}$.

As $R_{f} \rightarrow R_{+}$and $t_{f}>0$ along with $\left.D_{-}\right|_{i}>0$, we see that, $t_{f} \rightarrow \infty$. So, the negative sign for $R(t)$ describes a collapsing model in the incipient limit. Equation (26) also shows that for an asymptotic observer, the formation of the event horizon takes infinite time implying that the event horizon is an infinite red shifted surface, which matches with the classical result, as stated earlier while choosing the $\mathrm{RN}$ coordinates.

\section{Action for the scalar field $\Phi$}

The action for the scalar field $\Phi$ is written as a sum of the actions,

$$
\begin{aligned}
S_{\Phi}= & \left.\left.S_{\Phi}\right)_{\text {in }}+S_{\Phi}\right)_{\text {out }} \\
= & 2 \pi \int d t\left[-\left(\partial_{t} \Phi\right)^{2}\left(\int_{0}^{R} d r r^{2} \frac{1}{T_{t}}\right)\right] \\
& +2 \pi \int d t\left[\left(\partial_{r} \Phi\right)^{2}\left(\int_{0}^{R} d r r^{2} T_{t}\right)\right] \\
& +2 \pi \int d t\left[-\left(\partial_{t} \Phi\right)^{2}\left(\int_{R}^{\infty} d r r^{2} \frac{1}{\left.\left.1-\frac{2 M}{r}+\frac{Q^{2}}{r^{2}}\right)\right]}\right.\right. \\
& +2 \pi \int d t\left[\left(\partial_{r} \Phi\right)^{2}\left(\int_{R}^{\infty} d r r^{2}\left(1-\frac{2 M}{r}+\frac{Q^{2}}{r^{2}}\right)\right)\right],
\end{aligned}
$$

where the limits of the integration w.r.t. $r$ for $\left.S_{\Phi}\right)_{i n}$ are from 0 to $R$ while for $\left.S_{\Phi}\right)_{\text {out }}$ are from $R$ to $\infty$.

$T_{t} \rightarrow D$ (upto leading order) as $R \rightarrow R_{H}$ [from $e q^{n}$ (24)]. So,

$\lim _{R \rightarrow R_{H}} \frac{T_{t}}{1-\frac{2 M}{r}+\frac{Q^{2}}{r^{2}}}=\frac{R^{2}-2 M R+Q^{2}}{r^{2}-2 M r+Q^{2}} \frac{r^{2}}{R^{2}}=0$.

$T_{t}$ vanishes faster than $\left(1-\frac{2 M}{r}+\frac{Q^{2}}{r^{2}}\right)$ in the limit $R \rightarrow$ $R_{H}$. Thus, for coefficients of $-\left(\partial_{t} \Phi\right)^{2}$, the $\frac{1}{T_{t}}$ term dominates 
and for coefficients of $\left(\partial_{r} \Phi\right)^{2}$, the term which dominates is $\left(1-\frac{2 M}{r}+\frac{Q^{2}}{r^{2}}\right)$. Thus, in the incipient limit,

$$
\begin{aligned}
S_{\Phi} \rightarrow & 2 \pi \int d t\left[-\frac{1}{D} \int_{0}^{R_{H}} d r r^{2}\left(\partial_{t} \Phi\right)^{2}\right] \\
& +2 \pi \int d t\left[\int_{R_{H}}^{\infty} d r r^{2}\left(1-\frac{2 M}{r}+\frac{Q^{2}}{r^{2}}\right)\left(\partial_{r} \Phi\right)^{2}\right] .
\end{aligned}
$$

\section{Mode expansion for $\Phi$}

For a massless scalar field $\Phi$, one can easily check from its equation of motion, that is $\partial^{2} \Phi=0$, that for $r<R(t)$ (from $\left.\left.S_{\Phi}\right)_{i n}\right)$

$\frac{\partial^{2} \Phi}{\partial r^{2}}+\frac{2}{r} \frac{\partial \Phi}{\partial r}=\frac{1}{T_{t}^{2}} \frac{\partial^{2} \Phi}{\partial t^{2}}-\frac{T_{t}}{T_{t}^{3}} \frac{\partial \Phi}{\partial t}$,

where, $T_{t}$, and hence its powers and derivatives w.r.t. $t$, are independent of $r$.

Similarly, for $r>R(t)$, we have [from $\left.S_{\Phi}\right)_{\text {out }}$ )],

$$
\begin{aligned}
& \left(1-\frac{2 M}{r}+\frac{Q^{2}}{r^{2}}\right)^{2} \frac{\partial^{2} \Phi}{\partial r^{2}} \\
& +\frac{2(r-M)}{r^{2}}\left(1-\frac{2 M}{r}+\frac{Q^{2}}{r^{2}}\right) \frac{\partial \Phi}{\partial r}=\frac{\partial^{2} \Phi}{\partial t^{2}}
\end{aligned}
$$

From $e q^{n}$ (29) and $e q^{n}$ (30), we have the following mode expansion (due to the separability property of the above equations),

$\Phi(r, t)=\sum_{k} a_{k}(t) f_{k}(r)$

where $a_{k}(t)$ are the modes and $f_{k}(r)$ are real-valued smooth functions of $r$.

$S_{\Phi}$ in terms of modes $a_{k}$ is (as $\left.R \rightarrow R_{H}\right)$,

$S_{\Phi}=\int d t \sum_{k, k^{\prime}}\left[-\frac{1}{2 D} \frac{d a_{k}}{d t} A_{k k^{\prime}} \frac{d a_{k^{\prime}}}{d t}+\frac{1}{2} a_{k} B_{k k^{\prime}} a_{k^{\prime}}\right]$,

with the following definitions for $A_{k k^{\prime}}$ and $B_{k k^{\prime}}$,

$$
\begin{aligned}
& A_{k k^{\prime}}:=4 \pi \int_{0}^{R_{H}} d r r^{2} f_{k}(r) f_{k^{\prime}}(r), \\
& B_{k k^{\prime}}:=4 \pi \int_{R_{H}}^{\infty} d r r^{2}\left(1-\frac{2 M}{r}+\frac{Q^{2}}{r^{2}}\right) f_{k}^{\prime}(r) f_{k^{\prime}}^{\prime}(r),
\end{aligned}
$$

where, $f_{k}^{\prime}(r):=\frac{\partial f_{k}(r)}{\partial r}$. Note that, both $A_{k k^{\prime}}$ and $B_{k k^{\prime}}$ are independent of $r$ and $t$ (as no $R(t)$ appears in them).

The cojugate momenta, $\pi_{k} \mathrm{~s}$ (to the modes $a_{k}$ ), are defined as,

$\pi_{k}:=\frac{\partial \mathcal{L}_{\Phi}}{\partial \dot{a}_{k}} \equiv-i \frac{\partial}{\partial a_{k}}$,

where, $\dot{a}_{k}:=\frac{d a_{k}}{d t}$, and from $e q^{n}$ (32), we have (with $\mathcal{L}_{\Phi}$ defined as the Langrangian for $\Phi)$,

$$
\begin{aligned}
& \mathcal{L}_{\Phi}=\sum_{k, k^{\prime}}\left[-\frac{1}{2 D} \dot{a}_{k} A_{k k^{\prime}} \dot{a}_{k^{\prime}} d t+\frac{1}{2} a_{k} B_{k k^{\prime}} a_{k^{\prime}}\right], \\
& \mathcal{L}_{\Phi}=-\frac{1}{2 D}\left(\dot{\mathbf{a}}^{T} \mathbf{A} \dot{\mathbf{a}}\right)+\frac{1}{2}\left(\mathbf{a}^{T} \mathbf{B a}\right),
\end{aligned}
$$

where, $\mathbf{A}$ and $\mathbf{B}$ are non-singular linear operators, such that, $A_{k k^{\prime}} \in \mathbf{A}$ and $B_{k k^{\prime}} \in \mathbf{B}$ in the chosen bases, say $\left\{\dot{a}_{k}\right\}$ and $\left\{a_{k}\right\}$ respectively. In the basis $\left\{a_{k}\right\}, \mathbf{a}$ is a column vector, such that, $a_{k} \in \mathbf{a}$. One can express $\dot{\mathbf{a}}$ in a similar way in the basis $\left\{a_{k}\right\}$.

For the Hamiltonian of $\Phi, \mathcal{H}_{\Phi}$, we get,

$$
\begin{aligned}
\mathcal{H}_{\Phi} & =\sum_{k} \pi_{k} \dot{a}_{k}-\mathcal{L}_{\Phi} \\
& =\sum_{k, k^{\prime}}\left[\frac{1}{2 D} \dot{a}_{k} A_{k k^{\prime}} \dot{a}_{k^{\prime}} d t+\frac{1}{2} a_{k} B_{k k^{\prime}} a_{k^{\prime}}\right] \\
& =\frac{D}{2}\left(\Pi^{T} \mathbf{A}^{-1} \Pi\right)+\frac{1}{2}\left(\mathbf{a}^{T} \mathbf{B a}\right),
\end{aligned}
$$

where $\Pi$ is a column vector, such that, $\pi_{k} \in \Pi$, in a chosen basis say $\left\{\pi_{k}\right\}$ and $\mathbf{A}^{-1}$ denotes the inverse of $\mathbf{A}$.

$\mathbf{B}$ and $\mathbf{A}$ are real and symmetric infinite dimensional matrices and hence are self-adjoint. Thus, by the Spectral Theorem, there exists orthonormal bases of position space and momentum space consisting of respective eigenvectors of $\mathbf{B}$ and $\mathbf{A}$. Furthermore, all the eigenvalues are real. Say the bases for position space and momentum space are $\left\{b_{k}\right\}$ and $\left\{\dot{b}_{k}\right\}$ respectively (where, each $b_{k}$ is a linear combination of the original basis vectors $a_{k}$ and each $\dot{b}_{k}$ is a linear combination of the original basis vectors $\dot{a}_{k}$ ).

\section{The Schrödinger-like wave equation}

If we analyze the equation for one eigenvector $b \in\left\{b_{k}\right\}$, then our conclusion will be the same for all other eigenvectors (see [8]). So, we will solve the Schrödinger-like wave equation for a wave functional $\Psi\left(\left\{b_{k}\right\}, t\right)$ (see Appendix), which by the above assumption of equivalence is now a wave function $\psi(b, t)$. Hence, $\psi(b, t) \equiv \Psi\left(\left\{b_{k}\right\}, t\right)$. Thus, using $e q^{n}$ (37), we write the Schrödinger-like wave equation (for a single 
eigenvector $b$ ) as,

$$
\begin{aligned}
& {\left[-\left(1-\frac{2 M}{R}+\frac{Q^{2}}{R^{2}}\right) \frac{1}{2 \alpha} \frac{\partial^{2}}{\partial b^{2}}+\frac{1}{2} \beta b^{2}\right] \psi(b, t)} \\
& =i \frac{\partial \psi(b, t)}{\partial t}
\end{aligned}
$$

where, $\alpha$ and $\beta$ are the eigenvalues of $\mathbf{A}$ and $\mathbf{B}$ respectively.

Let us define a new time parameter,

$\eta:=\int_{0}^{t} d t\left(1-\frac{2 M}{R}+\frac{Q^{2}}{R^{2}}\right)$

leading to, $\frac{\partial \eta}{\partial t}=D$,

and write $e q^{n}(40)$ as

$\left[-\frac{1}{2 \alpha} \frac{\partial^{2}}{\partial b^{2}}+\frac{\beta}{2 D} b^{2}\right] \psi(b, \eta)=i \frac{\partial \psi(b, \eta)}{\partial \eta}$.

Define,

$\omega^{2}(\eta):=\left(\frac{\beta}{\alpha}\right) \frac{1}{D}=: \frac{\omega_{0}^{2}}{D}$.

Then, $e q^{n}$ (43) becomes,

$\left[-\frac{1}{2 \alpha} \frac{\partial^{2}}{\partial b^{2}}+\frac{1}{2} \alpha \omega^{2}(\eta) b^{2}\right] \psi(b, \eta)=i \frac{\partial \psi(b, \eta)}{\partial \eta}$,

where, we have chosen to set $\eta(t=0)=0$. Observe that, $e q^{n}(45)$ is a time dependent Simple Harmonic Oscillator (SHO) equation with $\omega(\eta)$ as the SHO's frequency.

In the incipient limit [using $e q^{n}(25)$ and $e q^{n}(23)$ ],

$\frac{d D}{d t}=\left[D_{-} \frac{R_{+}}{R^{2}}+D_{+} \frac{R_{-}}{R^{2}}\right] \approx-\left.D_{-}\right|_{i} \frac{R_{+}}{R_{+}^{2}} D=-\frac{\left.D_{-}\right|_{i} D}{R_{+}}$

Integrating $e q^{n}$ (46) w.r.t. $t$ one gets (as $R \rightarrow R_{H}$ ),

$D=1-\frac{2 M}{R(t)}+\frac{Q^{2}}{R(t)^{2}} \sim e^{-\left.D_{-}\right|_{i} t / R_{+}}$.

From $e q^{n}$ (47) we see that at late time, $1-\frac{2 M}{R(t)}+\frac{Q^{2}}{R(t)^{2}} \sim$ $e^{-\left.D_{-}\right|_{i} t / R_{+}}$. Since we are interested in the incipient limit, that is, in late times of the collapsing process, we can choose the behaviour of $R(t)$ at early times as per our convenience for simplifying calculations. So we choose both past and future behaviour of $R(t)$ to be stationary. We can take the metric to be flat for all $t \in(-\infty, 0)$. Stationarity in future can be achieved by taking a cut-off time $t_{f}$ for the collapse and then allowing $t_{f} \rightarrow \infty$, thus going into the continual collapse case till black hole formation. Thus,

$D= \begin{cases}1, & \text { for } t \in(-\infty, 0) \\ e^{-\left.D_{-}\right|_{i} t / R_{+}}, & \text {for } t \in\left(0, t_{f}\right) \\ e^{-\left.D_{-}\right|_{i} t_{f} / R_{+}}, & \text {for } t \in\left(t_{f}, \infty\right)\end{cases}$

The above choice of $R(t)$ may seem problematic as $\frac{d R}{d t}$ is discontinuous at 0 and $t_{f}$, but Refs. [8,17] show that the particle production by the collapsing shell happens in the range, $0<t<t_{f}$ and in the $t_{f} \rightarrow \infty$ regime, all the solutions obtained are well-behaved. So with the above considerations, the wavefunction $\psi$ would capture the whole collapse scenario, and in the limit of $t_{f} \rightarrow \infty$ or $R(t) \rightarrow R_{H}$, black hole formation occurs.

We note that, at early times, $t \in(-\infty, 0)$, the spacetime is Minkowski and hence the initial vacuum states at $\mathcal{J}^{-}$ (past null infinity) are $\left({ }^{1}\right)$ just the simple harmonic oscillator ground states (this can be seen from the form of $e q^{n}(45)$, which with $\eta=0$, is the SHO equation). Thus,

$\psi_{0}(b):=\psi(b, \eta=0)=\left(\frac{\alpha \omega_{0}}{\pi}\right)^{1 / 4} e^{-m \omega_{0} b^{2} / 2}$

where $\psi_{0}(b)$ represents the SHO ground state and $\left\{\psi_{n}(b)\right\}$ will represent the $\mathrm{SHO}$ basis states at early times.

Equation (49) suggests that $\omega_{0}$ defined in $e q^{n}$ (44) is the ground state frequency associated with the initial vacuum state.

With the aid of $e q^{n}(49)$, the exact solution to $e q^{n}(45)$ is,

$\psi(b, \eta)=e^{i \chi(\eta)}\left[\frac{\alpha}{\pi \zeta^{2}}\right]^{1 / 4} \exp \left[i\left(\frac{\zeta_{\eta}}{\zeta}+\frac{i}{\zeta^{2}}\right) \frac{\alpha b^{2}}{2}\right]$,

where $\zeta$ is the solution of the equation,

$\zeta_{\eta \eta}+\omega^{2}(\eta) \zeta=\frac{1}{\zeta^{3}}$

with the following initial conditions,

$\zeta(0)=\frac{1}{\sqrt{\omega_{0}}}$,

$\zeta_{\eta}(0)=0$,

and, $\chi(\eta)$ is given by,

$\chi(\eta):=-\frac{1}{2} \int_{0}^{\eta} \frac{d \eta^{\prime}}{\zeta^{2}\left(\eta^{\prime}\right)}$.

\footnotetext{
${ }^{1}$ The intuition behind identifying the states at $\mathcal{J}^{-}$and states with $t \in$ $(-\infty, 0)$ comes from the fact that the observer is at $r \rightarrow \infty$ and at an early time at $t \rightarrow-\infty$ which is $\mathcal{J}^{-}$and at late times he is at $t \rightarrow \infty$ which is $\mathcal{J}^{+}$.
} 
Equations of the form $e q^{n}$ (45) have been extensively studied in Refs. [18-22].

From $e q^{n}$ (44), $e q^{n}$ (47) and $e q^{n}$ (48), we have (for $t>0$ ),

$\omega(\eta(t))=e^{\left.D_{-}\right|_{i} t / 2 R_{+}} \omega_{0}$.

Using $e q^{n}(42)$ and $e q^{n}(55)$,

$\Omega(t)=\left(\left.\frac{\partial \eta}{\partial t}\right|_{t>0}\right) \omega(\eta)=e^{-\left.D_{-}\right|_{i} t / 2 R_{+}} \omega_{0}$,

where $\Omega(t)$ is defined to be the frequency w.r.t. time $t$.

We note that at early times $\left(\mathcal{J}^{-}\right)$, the states are the initial vacuum states of SHO described by $\psi_{0}(b)$. With time, the frequency of the states $\Omega(t)$ evolve [as per $e q^{n}$ (56)] and more states get excited. Finally, when the observer measures them at $\mathcal{J}^{+}$(future null infinity), that is for some $t \in\left(t_{f}, \infty\right)$, we have (following the evolution as per the Schrödinger picture [23]),

$\psi(b, t)=\sum_{n} c_{n}(t) \phi_{n}(b)$,

where $c_{n}(t)$ are the probability amplitudes and the final SHO states $\left\{\phi_{n}(b)\right\}$ are with the frequency $\Omega_{f}=\Omega\left(t_{f}\right)$ (a constant), given by,

$\phi_{n}(b)=\left(\frac{\alpha \Omega_{f}}{\pi}\right)^{1 / 4} \frac{e^{-\alpha \Omega_{f} b^{2} / 2}}{\sqrt{2^{n} n !}} H_{n}\left(\sqrt{\alpha \Omega_{f}} b\right)$.

Here, $H_{n}$ are the Hermite polynomials. Note that,

$\Omega\left(t_{f}\right)=e^{-\left.D_{-}\right|_{i} t_{f} / 2 R_{+}} \omega_{0} ;$

$c_{n}$ can be computed from an overlap integral as (see Appendix),

$c_{n}= \begin{cases}\frac{(-1)^{n / 2} e^{i \chi}}{\left(\Omega_{f} \zeta^{2}\right)^{1 / 4}} \sqrt{\frac{2}{P}}\left(1-\frac{2}{P}\right)^{n / 2} \frac{(n-1) ! !}{\sqrt{n !}}, & \text { for even } n \\ 0, & \text { for odd } n,\end{cases}$

where $P:=1-\frac{i}{\Omega_{f}}\left(\frac{\zeta_{\eta}}{\zeta}+\frac{i}{\zeta^{2}}\right)$.

\section{Unitarity from density matrix}

We compute the density matrices, $\hat{\rho}_{i}$ and $\hat{\rho}_{f}$, for the initial $\left(\mathcal{J}^{-}\right)$and the final $\left(\mathcal{J}^{+}\right)$states respectively. We can write the $\hat{\rho}_{i}$ and $\hat{\rho}_{f}$ as (see $\left.[10,11]\right)$,

$\hat{\rho}_{i}=\sum_{m, n} l_{m} l_{n}^{*}\left|\psi_{m}\right\rangle\left\langle\psi_{n}\right|$, $\hat{\rho}_{f}=\sum_{m, n} c_{m} c_{n}^{*}\left|\phi_{m}\right\rangle\left\langle\phi_{n}\right|$,

where, $l_{n}$ and $c_{n}$ are the probability amplitudes appearing in the intial and final states respectively.

Since initially the system was in the SHO eigenstates $\left\{\psi_{n}\right\}$ and the wavefunction is normalized, we have,

$\operatorname{Tr}\left(\hat{\rho}_{i}\right)=1$.

From $e q^{n}(60)$, with $\kappa:=\left|1-\frac{2}{P}\right|$, one has

$$
\begin{aligned}
\operatorname{Tr}\left(\hat{\rho}_{f}\right) & =\sum_{\text {even } n}\left|c_{n}\right|^{2} \\
& =\frac{2}{\sqrt{\Omega_{f} \zeta^{2}}|P|} \sum_{\text {even } n} \frac{(n-1) ! !}{n !} \kappa^{n} \\
& =\frac{2}{\sqrt{\Omega_{f} \zeta^{2}}|P|} \frac{1}{\sqrt{1-\kappa^{2}}} \\
& =\frac{2}{\sqrt{\Omega_{f} \zeta^{2}}|P|} \frac{1}{\sqrt{1-\left|1-\frac{2}{P}\right|^{2}}} .
\end{aligned}
$$

$P$ has been computed explicitly and used in $e q^{n}(64)$ to obtain (see Appendix),

$\operatorname{Tr}\left(\hat{\rho}_{f}\right)=1$.

By $e q^{n}$ (65), we have shown that the necessary condition for the unitary evolution of states holds. For the sufficiency, we compute $\operatorname{Tr}\left(\hat{\rho}_{f}^{2}\right)$. From $e q^{n}(62)$,

$$
\hat{\rho}_{f}=\sum_{m, n} c_{m} c_{n}^{*}\left|\phi_{m}\right\rangle\left\langle\phi_{n}\right|
$$

leading to, $\hat{\rho}_{f}^{2}=\left(\sum_{m, n} c_{m} c_{n}^{*}\left|\phi_{m}\right\rangle\left\langle\phi_{n}\right|\right)\left(\sum_{i, j} c_{i} c_{j}^{*}\left|\phi_{i}\right\rangle\left\langle\phi_{j}\right|\right)$

$$
=\sum_{m, n, i, j} c_{m} c_{i} c_{n}^{*} c_{j}^{*}\left|\phi_{m}\right\rangle\left\langle\phi_{n} \mid \phi_{i}\right\rangle\left\langle\phi_{j}\right|
$$

$=\sum_{m, n, j} c_{m} c_{j}^{*}\left|c_{n}\right|^{2}\left|\phi_{m}\right\rangle\left\langle\phi_{j}\right|$

$=\sum_{m, j} c_{m} c_{j}^{*}\left|\phi_{m}\right\rangle\left\langle\phi_{j}\right|\left(\sum_{n}\left|c_{n}\right|^{2}\right)$

$=\sum_{m, j} c_{m} c_{j}^{*}\left|\phi_{m}\right\rangle\left\langle\phi_{j}\right|$

$\times\left(a s,\left(\sum_{n}\left|c_{n}\right|^{2}\right)=1\right.$ byeq $\left.q^{n}(65)\right)$

$=\hat{\rho}_{f}$. 
Thus, by $e q^{n}$ (66) we get,

$\operatorname{Tr}\left(\hat{\rho}_{f}^{2}\right)=\operatorname{Tr}\left(\hat{\rho}_{f}\right)=1$.

Analytically, the idempotency of the final density matrix holds indicating a pure state to pure state transition.

\section{Unitarity from conservation of probability}

The probability current 4 -vector $J^{\mu}$ is defined as,

$$
\begin{aligned}
& J^{0}=|\psi|^{2}, \\
& \mathbf{J}=\frac{1}{2 \alpha i}\left[\psi^{*} \nabla \psi-\psi \nabla \psi^{*}\right] .
\end{aligned}
$$

As $b$ is an eigenfunction of $\mathbf{B}$ which is independent of spatial coordinate $x^{i}$, we conclude that $\mathbf{J}=\mathbf{0}$. This suggests,

$\nabla_{\mu} J^{\mu}=\frac{\partial|\psi|^{2}}{\partial t_{o b s}}$

Writing $t_{o b s}=t$ (for the observer's time coordinate), we have (from equation (42)),

$\nabla_{\mu} J^{\mu}=\frac{\partial|\psi|^{2}}{\partial t}=\frac{\partial|\psi|^{2}}{\partial \eta} \frac{\partial \eta}{\partial t}=D \frac{\partial|\psi|^{2}}{\partial \eta}$

For, $R \rightarrow R_{H}, \nabla_{\mu} J^{\mu}=0 \quad($ as,$D \rightarrow 0)$

So, we have shown analytically $\left(e q^{n} 71\right)$, that probability is conserved in the system, in the incipient limit of black hole formation.

\section{Extremal case}

For the extremal case, $|Q|=M$. From $e q^{n}$ (23) for $R_{ \pm}=|Q|=M$ (the event horizon), we obtain the classical behaviour of the shell as,

$R_{f}=R_{ \pm}+\frac{1}{\left[\frac{t_{f}}{R_{ \pm}^{2}} \pm \frac{1}{R_{0}-R_{ \pm}}\right]}$

Like $e q^{n}$ (26), $e q^{n}$ (72) also suggests that classically, the collapsing shell is infinitely red-shifted for an asymptotic observer.

For the extremal case, for $R \rightarrow R_{H}$,

$$
R(t)=R_{ \pm}+\frac{1}{\left[\frac{t}{R_{ \pm}^{2}} \pm \frac{1}{R_{0}-R_{ \pm}}\right]},
$$

leading to, $D \sim \frac{1}{\left(\frac{t}{R_{ \pm}} \pm \frac{R_{ \pm}}{R_{0}-R_{ \pm}}\right)^{2}}$

Following previous arguments, here one has,

$$
D= \begin{cases}1, & \text { for } t \in(-\infty, 0) \\ \frac{1}{\left(\frac{t}{R_{ \pm}} \pm \frac{R_{ \pm}}{R_{0}-R_{ \pm}}\right)^{2}}, & \text { for } t \in\left(0, t_{f}\right) \\ \frac{1}{\left(\frac{t_{f}}{R_{ \pm}} \pm \frac{R_{ \pm}}{R_{0}-R_{ \pm}}\right)^{2}}, & \text { for } t \in\left(t_{f}, \infty\right) .\end{cases}
$$

The corresponding $\omega(\eta(t)), \Omega(t)$ and $\Omega\left(t_{f}\right)$ are

$$
\begin{aligned}
\omega(\eta(t)) & =\left(\frac{t}{R_{ \pm}} \pm \frac{R_{ \pm}}{R_{0}-R_{ \pm}}\right) \omega_{0} \\
\Omega(t) & =\left(\left.\frac{\partial \eta}{\partial t}\right|_{t>0}\right) \omega(\eta)=\frac{1}{\left(\frac{t}{R_{ \pm}} \pm \frac{R_{ \pm}}{R_{0}-R_{ \pm}}\right)} \omega_{0} \\
\Omega\left(t_{f}\right) & =\frac{1}{\left(\frac{t_{f}}{R_{ \pm}} \pm \frac{R_{ \pm}}{R_{0}-R_{ \pm}}\right)} \omega_{0} .
\end{aligned}
$$

The rest of the analysis is similar to the non-extremal case with the above frequencies replacing the previous ones in the corresponding expressions.

\section{Conclusion}

So it has now been shown analytically and comprehensively that the black hole radiation is processed with a unitary evolution. This is accomplished using both facets of unitarity, namely the density matrix consideration as well as the conservation of probability consideration.

The Schrödinger-like wave equations that we wrote look similar to a minisuperspace version of Wheeler-deWitt equations [24]. Interestingly, such equations have a present resurgence, in the context of issues regarding unitarity [25-27]. However, how the Wheeler-deWitt formalism brings leads to the preservation of unitariy in the models is an issue left to ponder.

Saini and Stojkovic [10] showed that black hole radiation is a unitary process, for a Schwarzchild black hole, from the density matrix consideration through numerical estimates. We worked with a more general metric, the $\mathrm{RN}$ metric, and the Schwarzchild results can be trivially recovered from this work.

The calculations on unitarity are all in the incipient limit, the limit of formation of the back hole. So it does not really take care of the complete black hole evaporation process. However, if unitarity is preserved in this limit, it should be valid at every instant of time. In fact, there is a claim by Wallace [3] that the information loss paradox is not related to the black hole evaporation, but rather to the formation and 
its existence, as addressed by the incipient limit (see also $[4,5,28])$.

In saying this, we further emphasize that, what we have shown in this paper is that black hole radiation is unitary even in a non-globally hyperbolic spacetime. This has significant implications to the resolution of the information loss paradox.

It should also be emphasized that this is the first time where unitarity of black hole radiation is checked for an RN spacetime, which is globally non-hyperbolic to start with Ref. [29], that is, even as a static spacetime.

Acknowledgements $\mathrm{AD}$ would like to thank the Department of Science and Technology, Government of India for providing the INSPIRE-SHE scholarship which helped immensely in this research work.

Data Availability Statement This manuscript has no associated data or the data will not be deposited. [Authors' comment: There is no data taken from any sources. The work is based on analytical calculations, and that is given in the main text, or in the appendix in the paper itself.]

Open Access This article is distributed under the terms of the Creative Commons Attribution 4.0 International License (http://creativecomm ons.org/licenses/by/4.0/), which permits unrestricted use, distribution, and reproduction in any medium, provided you give appropriate credit to the original author(s) and the source, provide a link to the Creative Commons license, and indicate if changes were made.

Funded by SCOAP ${ }^{3}$.

\section{Appendix}

Alternate motivation for $S_{\text {shell }}$

Here we present a different action than $S_{\text {shell }}$. We will call it $S_{\text {new }}$. We will further show that in the incipient limit it will give rise to $\mathcal{H}_{\text {shell }}$ and $\Pi_{\text {shell }}$. Since we know that the shell behaves like a relativistic particle, we define the new action to be,

$$
\begin{aligned}
S_{\text {new }}= & -\int d \tau M=-\int d T \frac{M}{T_{\tau}} \\
= & -4 \pi \sigma \int d T R^{2}\left[1-2 \pi \sigma R \sqrt{1-R_{T}^{2}}\right] \\
& -\int d T \frac{Q^{2}}{2 R} \sqrt{1-R_{T}^{2}}, \\
= & -4 \pi \sigma \int d t R^{2} \\
& \left.\times \sqrt{D-\frac{1-D}{D} R_{t}^{2}}-2 \pi \sigma R \sqrt{D-\frac{R_{t}^{2}}{D}}\right] \\
& -\int d t \frac{Q^{2}}{2 R} \sqrt{D-\frac{R_{t}^{2}}{D}} .
\end{aligned}
$$

Then,

$$
\begin{aligned}
\mathcal{L}_{\text {new }}= & -4 \pi \sigma R^{2}\left[\sqrt{D-\frac{1-D}{D} R_{t}^{2}}-2 \pi \sigma R \sqrt{D-\frac{R_{t}^{2}}{D}}\right] \\
- & \frac{Q^{2}}{2 R} \sqrt{D-\frac{R_{t}^{2}}{D}}, \\
\Pi_{\text {new }}= & \frac{\partial \mathcal{L}_{\text {new }}}{\partial R_{t}} \\
= & \frac{4 \pi \sigma R^{2} R_{t}}{\sqrt{D}}\left[\frac{1-D}{\sqrt{D^{2}-(1-D) R_{t}^{2}}}-\frac{2 \pi \sigma R}{\sqrt{D^{2}-R_{t}^{2}}}\right] \\
& +\frac{Q^{2}}{2 R} \frac{R_{t}}{\sqrt{D} \sqrt{D^{2}-R_{t}^{2}}}, \\
\mathcal{H}_{\text {new }}= & \Pi_{\text {new }} R_{t}-\mathcal{L}_{\text {new }} \\
= & 4 \pi \sigma D^{3 / 2} R^{2}\left[\frac{19)}{\sqrt{D^{2}-(1-D) R_{t}^{2}}}\right. \\
& +\frac{Q^{2}}{2 R} \frac{D^{3 / 2}}{\sqrt{D^{2}-R_{t}^{2}}} .
\end{aligned}
$$

Now in the incipient limit we have,

$\mathcal{H}_{\text {new }}=\frac{4 \pi D^{3 / 2} \mu R^{2}}{\sqrt{D^{2}-R_{t}^{2}}}$,

$\Pi_{\text {new }}=\frac{4 \pi \mu R^{2} R_{t}}{\sqrt{D} \sqrt{D^{2}-R_{t}^{2}}}$,

where, $\mu:=\sigma\left(1-2 \pi \sigma R_{H}+\frac{Q^{2}}{8 \pi \sigma R_{H}^{3}}\right)$. Observe that these are the exact same equations we had obtained before in this incipient limit.

Derivation of the Schrödinger-like wave equation from the Wheeler-deWitt equation

The Wheeler-deWitt equation for a closed universe to which our system belongs to is given by,

$H_{t o t} \Psi_{t o t}=0$

where $H_{t o t}$ is the total Hamiltonian given as,

$H_{t o t}=H_{s y s}+H_{o b s}$,

and $\Psi_{t o t}\left[X^{\mu}, g_{\mu \nu}, \Phi, \mathcal{O}\right]$ is the total wavefunctional with all the constituents of the system including the observer's degrees of freedom denoted by $\mathcal{O}$. Note that the wavefunctional $\Psi_{t o t}$ is a functional only of the fields. 
We make an assumption that any weak interactions between the observer and the shell-metric-scalar system is contained in $H_{\text {sys }}$. Now this, along with the assumption that evolution of the shell-metric-scalar system and that the observer are independent of each other, we can argue that the total wavefunctional is separable and can be written as a sum over eigenstates as,

$\Psi_{t o t}=\sum_{k} c_{k} \Psi_{s y s}^{k}\left(s y s, t_{o b s}\right) \Psi_{o b s}^{k}\left(\mathcal{O}, t_{o b s}\right)$,

where $k$ labels the eigenstates $c_{k}$ 's are complex coefficients and $t_{o b s}$ is the observer's time coordinate.

Based on the above assumptions, we can argue that the observer will have his/her own evolution independent of the dynamics of the system and hence, his/her wavefunction $\Psi_{o b s}^{k}\left(\mathcal{O}, t_{o b s}\right)$ is assumed to satisfy the usual Schrödingerlike wave equation given as,

$H_{o b s} \Psi_{o b s}^{k}=i \frac{\partial \Psi_{o b s}^{k}}{\partial t_{o b s}}$.

Now let us go back to $e q^{n}(84)$, which implies,

$$
\sum_{k} c_{k}\left(H_{s y s}+H_{o b s}\right) \Psi_{s y s}^{k} \Psi_{o b s}^{k}=0,
$$

leading to, $\sum_{k}^{k} c_{k}\left(H_{s y s} \Psi_{s y s}^{k} \Psi_{o b s}^{k}+H_{o b s} \Psi_{o b s}^{k} \Psi_{s y s}^{k}\right)=0$,

leading to, $\sum_{k} c_{k}\left(H_{s y s} \Psi_{s y s}^{k} \Psi_{o b s}^{k}\right)=-\sum_{k} c_{k}\left(i \frac{\partial \Psi_{o b s}^{k}}{\partial t_{o b s}} \Psi_{s y s}^{k}\right)$

(by eq $\left.q^{n}(87)\right)$.

Now integrating the above equation w.r.t. $t_{o b s}$ gives,

$$
\begin{aligned}
\sum_{k} c_{k} & {\left[\int_{0}^{\infty} d t_{o b s}\left(H_{s y s} \Psi_{s y s}^{k} \Psi_{o b s}^{k}\right)\right] } \\
= & -\sum_{k} c_{k}\left[\int_{0}^{\infty} d t_{o b s}\left(i \frac{\partial \Psi_{o b s}^{k}}{\partial t_{o b s}} \Psi_{s y s}^{k}\right)\right], \\
= & -\sum_{k} c_{k}\left[\left.\left(\Psi_{s y s}^{k} \Psi_{o b s}^{k}\right)\right|_{0} ^{\infty}-\int_{0}^{\infty} d t_{o b s}\left(i \frac{\partial \Psi_{s y s}^{k}}{\partial t_{o b s}} \Psi_{o b s}^{k}\right)\right], \\
= & -\left.\Psi_{t o t}\right|_{0} ^{\infty}+\sum_{k} c_{k}\left[\int_{0}^{\infty} d t_{o b s}\left(i \frac{\partial \Psi_{s y s}^{k}}{\partial t_{o b s}} \Psi_{o b s}^{k}\right)\right] \\
& \left(\text { by eq } q^{n}(86)\right), \\
= & \sum_{k} c_{k}\left[\int_{0}^{\infty} d t_{o b s}\left(i \frac{\partial \Psi_{s y s}^{k}}{\partial t_{o b s}} \Psi_{o b s}^{k}\right)\right] \\
& \left(\text { as } \Psi \text { is } t_{o b s}-\text { independent }\right) .
\end{aligned}
$$

Above equation implies,

$$
\sum_{k} c_{k}\left[\int_{0}^{\infty} d t_{o b s}\left(H_{s y s} \Psi_{s y s}^{k}-i \frac{\partial \Psi_{s y s}^{k}}{\partial t_{o b s}}\right) \Psi_{o b s}^{k}\right]=0 .
$$

Then for arbitrary states $\Psi_{k}^{s y s}$ and since $c_{k}$ 's are independent of each other, $e q^{n}$ (88) can only hold if the integrand is zero but since $\Psi_{o b s}^{k}$ is not identically zero for all $k$. This implies,

$H_{s y s} \Psi_{s y s}^{k}=i \frac{\partial \Psi_{s y s}^{k}}{\partial t_{o b s}}$

Thus, the shell-metric-scalar system's wavefunctional $\Psi_{s y s}^{k}$ also satisfies its own Schrödinger-like wave equation. We can neglect the subscript sys and the superscript sys and would write $e q^{n}(89)$ as,

$H \Psi=i \frac{\partial \Psi}{\partial t_{o b s}}$,

where $H$ is the Hamiltonian and $\Psi$ is the wavefunctional of the shell-metric-scalar system.

Following usual minisuperspace arguments, we can truncate the field degrees of freedom to a finite subset and could consider the minisuperspace version of the Wheeler-deWitt equation. This truncation is useful and in the process, we do not lose any useful inputs of the system as long as we keep all the field degrees of freedom important to the analysis. So, since the shell exhibits spherical symmetry by assumption, all fields are assumed to respect spherical symmetry which is a reasonable assumption. So, the shell is described only by the radial degree of freedom denoted by $R\left(t_{o b s}\right)$.

Since we are working in the semi-classical regime, which means we are only interested in the quantum effects resulting from the quantization of the scalar field $\Phi$ in the presence of a classical background metric of the shell (where the shell is treated classically), without loss of generality, we can say that, $\mathcal{H}_{\Phi}$ (Hamiltonian for the massless scalar field) is the total Hamiltonian of the system, i.e., $\mathcal{H}_{\Phi} \equiv \mathcal{H}_{\text {sys }}$.

So for an asymptotic observer, $e q^{n}(90)$ reduces to,

$\mathcal{H}_{\Phi} \Psi_{\Phi}=i \frac{\partial \Psi_{\Phi}}{\partial t}$

Note that in $e q^{n}(91), \Psi_{\Phi}$ is a wavefunctional which we will be solving for, but this will be equivalent to solving a time-dependent Schrödinger equation for a wavefunction, $\Psi\left(\left\{a_{k}\right\}, t\right) \equiv \Psi_{\Phi}$, which is dependent on a set of infinite variables $\left\{a_{k}\right\}$ (the modes) and $t$. Furthermore, it belongs to an infinite dimensional Hilbert space.

\section{Computation of $c_{n}$}

Let us compute the $c_{n}$ 's explicitly. We know that,

$\psi(b, t)=\sum_{n} c_{n}(t) \phi_{n}(b)$, 
So, from the overlap integral we have,

$$
\begin{aligned}
c_{n}= & \int d b \phi_{n}^{*} \psi=\left(\frac{\alpha^{2} \Omega_{f}}{\pi^{2} \zeta^{2}}\right)^{1 / 4} \frac{e^{i \chi(\eta)}}{\sqrt{2^{n} n !}} \\
& \times \int d b \exp \left[-\frac{\alpha \Omega_{f} b^{2}}{2}+i\left(\frac{\zeta_{\eta}}{\zeta}+\frac{i}{\zeta^{2}}\right) \frac{\alpha b^{2}}{2}\right] \\
& \times H_{n}\left(\sqrt{\alpha \Omega_{f}} b\right) \\
= & \left(\frac{1}{\Omega_{f} \pi^{2} \zeta^{2}}\right)^{1 / 4} \frac{e^{i \chi(\eta)}}{\sqrt{2^{n} n !}} \\
& \times \int d x \exp \left[-\frac{x^{2}}{2}+\frac{x^{2}}{2} \frac{i}{\Omega_{f}}\left(\frac{\zeta_{\eta}}{\zeta}+\frac{i}{\zeta^{2}}\right)\right] H_{n}(x) \\
& \times\left(w i t h, x:=\sqrt{\alpha \Omega_{f}} b\right) \\
= & \left(\frac{1}{\Omega_{f} \pi^{2} \zeta^{2}}\right)^{1 / 4} \frac{e^{i \chi(\eta)}}{\sqrt{2^{n} n !}} \int d x e^{-P x^{2} / 2} H_{n}(x) \\
& \times\left(w i t h, P:=1-\frac{i}{\Omega_{f}}\left(\frac{\zeta_{\eta}}{\zeta}+\frac{i}{\zeta^{2}}\right)\right) \\
= & \left(\frac{1}{\Omega_{f} \pi^{2} \zeta^{2}}\right)^{1 / 4} \frac{e^{i \chi(\eta)}}{\sqrt{2^{n} n !}} I_{n} \\
& \times\left(w i t h, I_{n}:=\int d x e^{-P x^{2} / 2} H_{n}(x)\right)
\end{aligned}
$$

To compute $I_{n}$, let us consider the following generating function for the $H_{n}(x)$,

$J(z)=\int d x e^{-P x^{2} / 2} e^{-z^{2}+2 z x}=\sqrt{\frac{2 \pi}{P}} e^{-z^{2}(1-2 / P)}$,

since, $e^{-z^{2}+2 z x}=\sum_{n=0}^{\infty} \frac{z^{n}}{n !} H_{n}(x)$,

$$
\int d x e^{-P x^{2} / 2} H_{n}(x)=\left.\frac{d^{n}}{d z^{n}} J(z)\right|_{z=0},
$$

thus, $I_{n}=\sqrt{\frac{2 \pi}{P}}\left(1-\frac{2}{P}\right)^{n / 2} H_{n}(0)$,

as, $H_{n}(0)= \begin{cases}(-1)^{n / 2} \sqrt{2^{n} n !} \frac{(n-1) ! !}{\sqrt{n !}}, & \text { for even } n \\ 0, & \text { for odd } n .\end{cases}$

Thus we have,

$$
c_{n}= \begin{cases}\frac{(-1)^{n / 2} e^{i \chi}}{\left(\Omega_{f} \zeta^{2}\right)^{1 / 4}} \sqrt{\frac{2}{P}}\left(1-\frac{2}{P}\right)^{n / 2} \frac{(n-1) ! !}{\sqrt{n !}}, & \text { for even } n \\ 0, & \text { for odd } n .\end{cases}
$$

\section{Explicit computation of $\operatorname{Tr}\left(\hat{\rho}_{f}\right)$}

We know that,

$$
\operatorname{Tr}\left(\hat{\rho}_{f}\right)=\frac{2}{\sqrt{\Omega_{f} \zeta^{2}}|P|} \frac{1}{\sqrt{1-\left|1-\frac{2}{P}\right|^{2}}} .
$$

To compute $P$ explicitly, let us give the solution of,

$\zeta_{\eta \eta}+\omega^{2}(\eta) \zeta=\frac{1}{\zeta^{3}}$

as,

$\zeta=\frac{1}{\sqrt{\omega_{0}}} \sqrt{\epsilon^{2}+\varepsilon^{2}}$,

$\zeta_{\eta}=\frac{1}{\omega_{0} \zeta}\left(\epsilon \epsilon_{\eta}+\varepsilon \varepsilon_{\eta}\right)$,

where in terms of Bessel's functions, we have,

$\epsilon=\frac{\pi u_{0}}{2}\left[Y_{0}\left(2 \omega_{0}\right) J_{1}\left(u_{0}\right)-J_{0}\left(2 \omega_{0}\right) Y_{1}\left(u_{0}\right)\right]$,

$\varepsilon=\frac{\pi u_{0}}{2}\left[Y_{1}\left(2 \omega_{0}\right) J_{1}\left(u_{0}\right)-J_{1}\left(2 \omega_{0}\right) Y_{1}\left(u_{0}\right)\right]$,

$\epsilon_{\eta}=-\pi \omega_{0}^{2}\left[Y_{0}\left(2 \omega_{0}\right) J_{0}\left(u_{0}\right)-J_{0}\left(2 \omega_{0}\right) Y_{0}\left(u_{0}\right)\right]$,

$\varepsilon_{\eta}=-\pi \omega_{0}^{2}\left[Y_{1}\left(2 \omega_{0}\right) J_{0}\left(u_{0}\right)-J_{1}\left(2 \omega_{0}\right) Y_{0}\left(u_{0}\right)\right]$,

where, $u_{0}:=2 \omega_{0} \sqrt{1-\eta}$.

Now, substituting the definition of $P\left(e q^{n}(94)\right)$ in $e q^{n}$ (97), we have (using Mathematica),

$\operatorname{Tr}\left(\hat{\rho}_{f}\right)=\frac{\left|\zeta^{2} \Omega_{f}\right|}{\sqrt{\zeta^{2} \Omega_{f}} \sqrt{-\Im\left[\zeta^{2} \Omega_{f}\right] \Re\left[\zeta \zeta_{\eta}\right]+\left(1+\Im\left[\zeta \zeta_{\eta}\right]\right) \Re\left[\zeta^{2} \Omega_{f}\right]}}$.

Now, as $\Omega_{f}, \zeta$ and $\zeta_{\eta}$ are real [as is evident from $e q^{n} s$ (99) to (104)], we get from $e q^{n}(105)$,

$\operatorname{Tr}\left(\hat{\rho}_{f}\right)=1$.

\section{References}

1. S.W. Hawking, Commun. Math. Phys. 43, 199 (1975)

2. S.W. Hawking, Phys. Rev. D 14, 2460 (1976)

3. D. Wallace. arXiv:1710.03783 [gr-qc] (2017)

4. S.D. Mathur, Class. Quant. Grav. 26, 224001 (2009)

5. J. Polchinski. arXiv:1609.04036 [hep-th] (2016)

6. W.G. Unruh, R.M. Wald, Phys. Rev. D 52, 2176 (1995)

7. W.G. Unruh, R.M. Wald, Rep. Prog. Phys. 80, 092002 (2017)

8. T. Vachaspati, D. Stojkovic, L.M. Krauss, Phys. Rev. D 76, 024005 (2007)

9. T. Vachaspati, Class. Quant. Grav. 26, 215007 (2009)

10. A. Saini, D. Stojkovic, Phys. Rev. Lett. 114, 111301 (2015)

11. A. Saini, D. Stojkovic, Phys. Rev. D 97, 025020 (2018)

12. G. Birkhoff, R. Langer, Relativity and Modern Physics (Harvard University Press, Boston, 1923)

13. M. Heusler, Black Hole Uniqueness Theorems (Cambridge University Press, Cambridge, 1996)

14. W. Israel, Nuovo Cimento 44B, 1 (1966)

15. C.A. López, Phys. Rev. D 38, 3662 (1988)

16. J.E. Wang, E. Greenwood, D. Stojkovic, Phys. Rev. D 80, 124027 (2009)

17. E. Greenwood, JCAP 1001, 002 (2010) 
18. C.M.A. Dantas, I.A. Pedrosa, B. Baseia, Phys. Rev. A 45, 1320

24. B.S. DeWitt, Phys. Rev. 160, 1113 (1967) (1992)

19. H.R. Lewis, J. Math. Phys. 9, 1976 (1968)

20. H.R. Lewis, W.B. Riesenfeld, J. Math. Phys. 10, 1458 (1969)

21. I.A. Pedrosa, J. Math. Phys. 28, 2662 (1987)

22. M. Kolopanis, T. Vachaspati, Phys. Rev. D 87, 085041 (2013)

25. S. Pal, N. Banerjee, Phys. Rev. D 90, 104001 (2014)

26. S. Pal, N. Banerjee, Phys. Rev. D 91, 044042 (2015)

27. S. Pal, N. Banerjee, J. Math. Phys. 57, 122502 (2016)

28. D.N. Page, Phys. Rev. Lett. 71, 3743-3746 (1993)

23. J.J. Sakurai, J. Napolitano, Modern Quantum Mechanics, 2nd edn. (Pearson Education Limited, 2014) 